\title{
Rethinking routine airline testing during COVID-19
}

To the editor - Designing national testing policies for SARS-CoV-2 faces several challenges, both technical and political. Even the choice of testing technology is difficult. Reverse transcription polymerase chain reaction (RT-PCR) tests are - given their superior analytic sensitivity - ideal for confirming the presence of SARS-CoV-2 (ref. ${ }^{1}$ ). However, the requisite specialized equipment, skilled labour and time makes the administration of RT-PCR tests tedious. Rapid antigen tests offer less cumbersome administration, but forfeit accuracy ${ }^{2}$. False negatives are more likely with rapid antigen tests compared to RT-PCR tests, although both may be subject to error. Test superiority cannot be equated with test accuracy, given that viral load varies over the course of an infection ${ }^{3,4}$.

As an alternative to reliance on one-off imperfect tests, considering testing frequency in addition to testing sensitivity can address concerns over false negatives. How well viral molecules can be detected in a single sample matters, but so does how effectively infections can be detected at the population level through repeated testing within an overall testing strategy ${ }^{1}$. The gold standard for such an approach combines superior analytic sensitivity with high testing frequency. This approach is particularly useful in the case of testing for international travel, for which countries must balance competing pressures - such as the need to reduce infections and the need to facilitate movement of people and goods. As of January 2022, Canada, Jordan and Rwanda have adopted this approach: unencumbered entry to these countries requires negative SARS-CoV-2 test results from inbound travellers before and after arrival.

Challenges nevertheless persist. False negatives are - despite high test frequency - still possible should testing occur (1) in close succession or (2) too far apart. An infected traveller who lacks sufficient viral load is likely to test negative before arrival. However, this individual will also (incorrectly) test negative after arrival if the tests are administered too close together to allow enough time for a measurable increase in viral load. Inordinately large spacing between successive tests yields similar results. Test frequency matters, but so too does when tests are administered.
In addition to minimizing the risk of false negatives, testing programmes must consider additional factors such as accessibility and broader economic implications. One key consideration is pricing structure. A high demand for rapid SARS-CoV-2 test results imposes costs that are directly borne by consumers during test administration ${ }^{5}$. Relaxing the testing window can assuage cost concerns. Affording choice over where and when to test alleviates the price premium associated with rapid point-of-engagement testing. However, near-term savings realized through relaxed testing windows risks false negatives. Viral load during testing may be low, or infection may follow test administration but precede community engagement ${ }^{6}$.

Moving forward, governments should consider subsidizing targeted, rapid, point-of-engagement testing. This is particularly timely for air travel, access to which necessitates - since the SARS-CoV-2 outbreak - adherence to stringent government-imposed testing requirements. Entry into Australia, France or the USA requires administration of a rapid antigen test $24 \mathrm{~h}$ before trip commencement. Stringency imposes a price premium that is unlikely to be covered by insurers ${ }^{5}$. This structure dis-incentivizes passengers from flying, creating macroeconomic ripple effects. Aviation is a key enabler of numerous other economic activities and social relationships.

Inhibition of these activities owing to SARS-CoV-2 containment measures should be addressed. Subsidized SARS-CoV-2 testing for passengers can facilitate this outcome. For countries aiming to maximize border-testing efficiency and with the capacity to do so, public subsidies are probably needed in the short term to create economies of scale in testing. Although these expenditures are likely to decline in time, a revenue-neutral approach for SARS-CoV-2 travel testing deserves consideration, given deficit spending concerns. This may be achieved by subsidizing the administration of SARS-CoV-2 test at airports before versus after arrival.

Vaccination status should have a role in determining subsidy eligibility and magnitude. Vaccine mandates have proven effective in raising vaccination rates, although their legality remains unclear ${ }^{7,8}$. The provision of government-funded vaccine incentives faces less legal scrutiny. Underwriting rapid, point-of-engagement testing costs for vaccinated individuals while withholding (or offering less generous coverage) for unvaccinated individuals is a timely policy lever - one that recognizes individual rights to vaccinate (or not), while conveying that exercising these rights carries consequences ${ }^{8}$. Tying subsidy provision to vaccination reflects the effectiveness of vaccination in reducing infection ${ }^{9,10}$.

Limiting SARS-CoV-2 transmission requires the timely identification of infection. Where air travel is concerned, test administration should - in the interests of evidence-informed health policy - occur as close to the actual travel as possible. Yet as with any public policy, such a policy is an imperfect compromise. Trade-offs between test stringency, test frequency, turnaround time and the ensuing burdens imposed on the public deserves consideration by policy-makers. Defeating SARS-CoV-2 entails robust health interventions that consider the importance of unimpeded social and economic activity. We need both, in equal measure.

\section{Ashley Nunes ${ }^{1 凶}$ and Prabhat Jha ${ }^{2}$ \\ ${ }^{1}$ Harvard Law School, Cambridge, MA, USA. ${ }^{2}$ Centre for Global Health Research, Unity Health Toronto and University of Toronto, Toronto, Ontario, Canada.

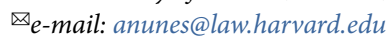

Published online: 21 February 2022 https://doi.org/10.1038/s41562-022-01317-7

References

1. Mina, M. J., Parker, R. \& Larremore, D. B. N. Engl. J. Med. 383, el20 (2020).

2. Service, R. F. Coronavirus antigen tests: quick and cheap, but too often wrong? Science, https://go.nature.com/3JgfrOJ (May 2020).

3. Zou, L. et al. N. Engl. J. Med. 382, 1177-1179 (2020).

4. He, X. et al. Nat. Med. 26, 672-675 (2020).

5. Skytrax. The varying cost of a PCR test around the world. Skytrax, https://go.nature.com/34IpA7z (April 2021).

6. Cevik, M. et al. Lancet Microbe 2, e13-e22 (2021).

7. Mills, M. C. \& Rüttenauer, T. Lancet Public Health 7 , e15-e22 (2022).

8. Jacobson V. Massachusetts, 197 U.S. 11, https://go.nature. com/3GFeLAt (1905).

9. Singanayagan, A. et al. Lancet Infect. Dis. 22, 183-195 (2022).

10. Government of Canada. COVID-19: Summary data about travellers, testing and compliance. canada.ca, https://go.nature. com/3GCfUJf (January 2022).

Competing interests

The authors declare no competing interests. 